\title{
Specialized morphology, not relatively large head size, facilitates competition between a small-bodied specialist and large-bodied generalist competitors
}

\author{
J. M. Barends (iD \& B. Maritz \\ Department of Biodiversity and Conservation Biology, University of the Western Cape, Cape Town, South Africa
}

\begin{abstract}
Keywords
body size; head morphology; interspecific competition; phenotypic specialization; snakes; specialists vs. generalists.
\end{abstract}

\section{Correspondence \\ Jody Michael Barends, Department of \\ Biodiversity and Conservation Biology, University of the Western Cape, Private Bag X17, Bellville, \\ Cape Town 7545, South Africa. \\ Email: Jbarends99@gmail.com}

Editor: Anthony Herrel

Associate Editor: Donald Miles

Received 22 April 2020; revised 4 May 2021; accepted 7 June 2021

doi:10.1111/jzo.12914

\begin{abstract}
Interspecific competition for limited resources should theoretically occur between species that are morphologically similar to each other. Consequently, species that reduce competition by adapting to specialize on a specific resource should be morphologically disparate to sympatric contemporaries and show evidence of phenotypic specialization. However, few studies have compared the morphologies of specialist and generalist competitors. In this context, we compare the feeding morphology and diet of an obligate, specialist, bird-egg-eating snake to three sympatric generalists that only facultatively consume bird eggs. We measured and compared body and head morphology of preserved museum specimens of each of four, syntopic snake species from southern Africa: the obligate bird-egg-eating rhombic egg-eater (Dasypeltis scabra), and the facultative bird-egg-eating boomslang (Dispholidus typus), cape cobra (Naja nivea) and mole snake (Pseudaspis cana). Given the physical challenges of consuming bird eggs in snakes, we predicted that consumption of bird eggs would be facilitated by the evolution of relatively larger heads in the smaller-bodied Dasypeltis. We found that head size was not phylogenetically conserved in the clades of these taxa and that contrary to our expectations, the specialist egg-eaters evolved to possess significantly smaller heads relative to body size than their competitors. We found a positive relationship between dietary niche breadth and head size within these species and their close relatives. Thus, relatively large-headed species have evolved diverse diets that overlap with the restricted diets of the small-headed specialist thereby producing this atypical competitive interaction. Our findings suggest that specialized adaptations can decouple typical body-size-constrained competition dynamics between sympatric snake species and highlight the complexity of the origins of dietary specialization.
\end{abstract}

\section{Introduction}

Sympatric species that are ecologically and morphologically similar to each other are predicted to compete for shared resources (Toft, 1985; Arthur, 1987; Ye, Yang \& Liang, 2019). This competition can result in selection for differences in ecology and morphology that act to reduce competition between such species. As a consequence, species that specialize on a narrow range of the available resources to reduce interspecific competition by efficiently exploiting a specific niche often have unique adaptive morphology that differentiates them from sympatric competitors (Schoener, 1974; Pianka, 1978; Mori \& Vincent, 2008). Accordingly, studying the links between phenotypic specialization and niche utilization across interspecific competitors has the potential to elucidate the selective pressures behind factors like resource partitioning that have ultimately driven biological diversification (Roughgarden, 1983; McGill et al., 2006). However, comparative studies that seek to quantify phenotypic specialization of ecological specialists relative to generalists are surprisingly sparse (Mori \& Vincent, 2008).

Generally, competition between species over shared resources can be predicted based on phenotypic similarities (Pianka, 1978; Schoener, 1983; McGill et al., 2006). For example, morphologically similar species of comparable body size are likely to compete with each other over specific resources because these species are likely to share similar ecological constraints (Bloch, Stevens \& Willig, 2011). As such, body size can act as an effective predictor for interspecific competition between similar organisms within biological communities comprised of a range of variably sized species (Case, Faaborg, \& Sidell, 1983; Alatalo \& Moreno, 1987; LaBarbera, 1989; Robertson, 1998; Luiselli, 2006). Additionally, body size can also act as a predictor for competitive superiority between competing species (Travis, 1980; Schoener, 1983; Persson, 1985; Morin \& Johnson, 1988; Ramsay, Kaiser \& Hughes, 1997; Zeng \& Lu, 2009; Nakayama \& Fuiman, 2010). Typically, larger-bodied species are competitively superior to 
smaller-bodied species in interference competition but smallerbodied species are more efficient at exploitation competition (Alatalo \& Moreno, 1987; Nascimento et al., 2011). As a result, body size can be strongly linked to dynamics of competition and resource partitioning and potentially play an important role within phenotypic specialization of competing species.

Snakes represent an ideal group of terrestrial vertebrate predators in which to empirically test hypotheses relating to adaptive morphological differences between dietary specialists and generalists. Snakes are restricted in terms of the prey that they can consume as they are unable to mechanically reduce the sizes and process their captured prey (Greene, 1997; Mori \& Vincent, 2008), thereby making them gape-limited predators. As such, snake gape size, and therefore head size, can be directly linked to the range of prey that snakes consume (Arnold, 1993; Cundall \& Greene, 2000; Cundall, 2019), thus allowing for the inference of direct relationships between changes in the functional morphology of snake feeding apparatus and the consumption of specific prey (Rodríguez-Robles, Bell \& Greene, 1999; Vincent et al., 2006; Moon et al., 2019). For example, North American natricine snakes that feed exclusively on fish have longer quadrate bones and increased swallowing performance of large fish prey to congeneric generalists (Vincent et al., 2009).

Snakes that consume large prey are typically able to do so because they possess specialized adaptive morphology, or, are simply large-bodied and have large heads (Cundall \& Greene, 2000; Moon et al., 2019). Generally, snake species with larger heads can consume a broader range of prey than those with smaller heads (Arnold, 1993; Greene, 1997; Cundall \& Greene, 2000). As a result, large-bodied snakes are predicted to have a wide dietary niche (Shine, 1991; Arnold, 1993; Luiselli, 2006; Moon et al., 2019) and generalist diets. Conversely, dietary specialists that only consume a limited number of prey types should showcase predictable phenotypical adaptations in their head elements that facilitate the ingestion of their preferred prey (Mori \& Vincent, 2008).

As a source of food for snakes, bird eggs are generally infrequently utilized (de Queiroz \& Rodríguez-Robles, 2006; Gartner \& Greene, 2008) owing largely to the mechanical difficulties associated with bird egg consumption. Bird eggs are often large, hard, round and slippery (Tarboton, 2011; Bates \& Little, 2013), making them difficult for snakes to ingest using traditional prey transport mechanisms (Cundall \& Greene, 2000; Jayne, Voris \& Ng, 2018). Consequently, the majority of snake species that consume bird eggs are large-bodied generalist feeders with large gapes that allow them to circumvent the challenges of ingesting bird eggs (de Queiroz \& RodríguezRobles, 2006). As such, species that specialize on bird eggs should theoretically possess head and jaw elements that provide a gape size equal to or larger than generalist competitors of the same body size allowing them to better ingest and more efficiently exploit this mechanically challenging prey type. Although hypotheses relating to egg-eating performance have been tested between unrelated, allopatric species from different continents (Gartner \& Greene, 2008), they remain untested among specialist and generalist bird egg competitors within real-world snake communities.
African egg-eater snakes of the genus Dasypeltis represent a model group of species for comparative investigations relating to phenotypic specialization for feeding as all members of the genus are bird egg specialists (Branch, 1998; Alexander \& Marias, 2007; Bates \& Little, 2013). Occurring throughout subSaharan Africa and parts of the Arabian Peninsula, all members of Dasypeltis obligately feed only on bird eggs (Bates \& Little, 2013; Bates \& Broadley, 2018) and possess a host of unique morphological adaptations that functionally accommodate the exploitation of this prey type (Gans, 1952; Gans, 1974; Gartner \& Greene, 2008). Morphological adaptations in Dasypeltis include highly stretchable neck, jaw and mouth tissue, a buccal cavity devoid of teeth, as well as modified vertebral hypapophyses that allows them to ingest and crush bird eggs before regurgitating the shell remains (Gans, 1952; Broadley, 1990; Branch, 1998; Alexander \& Marias, 2007).

Somewhat surprisingly, members of Dasypeltis are not particularly large-bodied snakes (typically 160-1000 mm SVL; $\max$ SVL $=1061 \mathrm{~mm}$; see Bates \& Broadley, 2018) and are demonstrably smaller in absolute body size relative to competing snakes that also consume bird eggs. Southern African snakes are known consumers of bird eggs and occur sympatrically with Dasypeltis such as Cape cobras (Naja nivea; Layloo, Smith \& Maritz, 2017), boomslang (Dispholidus typus; Smith et al., 2019) and mole snakes (Pseudaspis cana; Underhill et al., 2009) each out-size rhombic egg-eaters in their respective absolute body size, with adults of each species reaching up to $2 \mathrm{~m}$ in total length (Alexander \& Marias, 2007). Given the obvious differences in absolute body size between Dasypeltis and their competitors, adult Dasypeltis are unlikely, in absolute terms, to have longer or wider heads than adults of generalist, facultative egg-eating competitors. However, Dasypeltis may have evolved larger heads (relative to their body size) to produce heads that allow them to compete with largerbodied competitors that possess absolutely larger heads.

We examined head size variation and its evolution among four, sympatric, bird-egg-eating snakes including the egg-eating specialist, Dasypeltis scabra and three egg-eating generalists: Naja nivea, Dispholidus typus and Pseudaspis cana. We hypothesized that D. scabra has experienced selection for larger head size, resulting in head sizes that are comparable to those of its larger-bodied, generalist competitors. We test this hypothesis by (1) quantifying and reconstructing head size across a phylogeny including our four target species, plus 30 other closely related species and (2) explicitly comparing head dimensions of our four target species. To provide context to our analysis, we additionally examine the relationship between dietary niche breadth and head size to better understand the evolutionary dynamics that characterize the interplay of dietary specialization, trophic morphology and interspecific competition in these snakes.

\section{Materials and methods}

\section{Phylogenetic coverage}

Because our four study species are highly divergent in their phylogenetic relatedness (Pyron, Burbrink \& Wiens, 2013; 
Zheng \& Wiens, 2016), differences in their morphologies and diet may reflect phylogenetic trends in the evolutionary histories of their respective clades rather than adaptive responses to ecological correlates like competition for food. We therefore sought to take common ancestry into account in our comparisons of these aspects. We increased our taxonomic coverage by collecting morphological and dietary data for our four study species and 30 additional snake taxa related to them, resulting in an expanded 34-taxon dataset (Table S1). These additional species comprised several closely related members of the Colubridae, Elapidae and Lamprophiidae sensu lato (including Pseudaspididae) families, respectively. We pruned the timecalibrated phylogeny of squamate reptiles presented in Zheng \& Wiens, (2016) to only included our selected taxa for use in our phylogenetic analyses (Fig. S1).

\section{Morphological measurements}

We measured the bodies and heads of preserved specimens of our study species (Dasypeltis scabra: $n=19$, Dispholidus typus: $n=15$, Naja nivea: $n=17$ and Pseudaspis cana: $n=14$ ), and the additional taxa comprising our expanded data set housed in herpetology collections at Iziko South African Museum in Cape Town, Bayworld Museum in Port Elizabeth and the University of the Western Cape (Table S1). We only included measurements from adult specimens and used equal numbers of male and female specimens as best as we could to minimize the effects of ontogenetic shifts and sexual size dimorphism on measurements. For each individual, we measured the following: snout-vent length (SVL), tail length, head height, head length (the distance between the posterior of the parietal scale to the snout), head width and lower jaw length (the distance between the dentary and the retroarticular process). See Vincent et al., (2006) for justification of the selection of these specific morphological measures concerning snake feeding apparatus. We measured SVL and tail length to the nearest $1 \mathrm{~mm}$ using a measuring tape and measured all head dimensions using digital calipers (accuracy: $0.001 \mathrm{~mm}$ ). Summarized measures of body and head sizes are presented in Table S2.

\section{Dietary niche}

To compare the relative importance of bird eggs within the diets of our study species and their relatives, we collated dietary information for each species from the literature and citizen science reports of feeding records published online. We estimated the consumption of different prey as a proportion for each snake species using the following prey categories: amphibians, birds, bird eggs, lizards, mammals, snakes and other prey. Using our estimated proportions of prey categories, we then calculated Levins' measure of niche breadth (Levins, 1968) based on the equation $B=1 / \sum p_{i}^{2}$ where $B$ is Levins' niche breadth and $p_{i}$ is the proportion of individuals consuming a particular prey type. We then standardized this measure to range between zero and one using the equation $B_{A}=(B-1)$ / $(n-1)$, where $B_{A}$ is Levin's standardized niche breadth, and $n$ is the total number of prey classes.

\section{Statistical analyses}

We performed all statistical analyses using SPSS v 23.3 (IBM Corp, 2017) and R software v 3.5.3 (R core team, 2020). To meet the assumptions of homoscedasticity and ensure that all data were normally distributed, we log-transformed all morphological body and head size measures.

To examine covariation within head measures and relevant PC axes independently of body sizes across our study species, we created a variance-covariance matrix of all head measures relative to body size for each species. We calculated this matrix by regressing all log-transformed head measures against log-transformed SVLs to generate size-adjusted residual contrast values for each specimen. We then input these sizeadjusted contrasts into a principal component analysis (PCA) using the prcomp function of the 'stats' $\mathrm{R}$ package ( $\mathrm{R}$ core team, 2020) to calculate the variance-covariance matrix. We used a scree plot and the broken stick method (Jackson, 1993) to identify significant principal component axes. The broken stick method compares the variances of each component and identifies those that explain more variance than expected as significantly important. We then averaged the values across all specimens of each species for each significant component.

To remove the effects of phylogeny on the above analysis, we also calculated a second matrix that took common ancestry into account using our expanded dataset. To create this phylogenetically corrected covariance matrix, we performed a phylogenetic PCA (pPCA) using the phyl.pca function of the 'phytools' R package (Revell, 2012). For this analysis, we used size-adjusted contrast values for our expanded 34-taxon dataset in conjunction with our phylogeny. We then identified significant PC axes and estimated relative head size for each species in a similar manner to the non-phylogenetically corrected PCA analysis.

To ensure that differences in head size between our study, species were not significantly driven by patterns of phylogenetic conservatism of head morphology among those species and their relatives, we tested for evidence of a phylogenetic signal in general head size (i.e. average scores derived from the main principal component, hereafter PC 1) across our expanded 34-taxon phylogeny. We tested for phylogenetic signal by calculating Blomberg's $K$ (Blomberg, Garland \& Ives, 2003). Blomberg's $K$ values lower than one indicate that traits of closely related species resemble each other less than expected under a Brownian motion model of evolution whereas $K$ values greater than one suggest stronger trait similarity between close relatives (Blomberg, Garland \& Ives, 2003).

We identified the most appropriate model of evolution for general head size across our phylogeny by comparing four evolutionary models, namely the Brownian motion, Early-burst, Ornstein-Uhlenbeck and white noise models of evolution using the 'geiger' R package (Pennell et al., 2014). We selected the most appropriate model based on each model's Akaike Information Criterion corrected for small sample size (AICc; Akaike, 1973) scores. We then visually demonstrated evolutionary divergences in head morphology between our study species and their relatives by performing a maximum likelihood ancestral reconstruction analysis of head size across our 
phylogeny. We used the anc.ML function of the 'phytools' $\mathrm{R}$ package, to reconstruct averaged PC 1 scores of each species across our phylogeny under the best performing model of evolution (see Results). We visualized these projections across the phylogeny using the contMap function of the same package.

To test the hypothesis that head size relative to body size differed between Dasypeltis scabra, Dispholidus typus, Naja nivea and Pseudaspis cana, we used a multivariate analysis of variance (MANOVA) to compare covariation in head sizes across these four species. For this analysis, we included the body size-adjusted measures of head height, head length, head width and lower jaw length, as well as the significant PC axes from our PCA analyses (i.e. PC 1, see Results below) and the dietary niche breadth of each species (Levins $B_{A}$ ) as the dependent variables, and we used sex and species as the grouping factors. We also included the associated Bonferroni post hoc pairwise comparisons for further examinations of differences of each variable between each combination of species (see Shine et al., 2006).

Lastly, we examined the relationship between relative head size and dietary specialization within our four study species. We did this by performing ordinary least squares regression analyses comparing average PC 1 estimates and Levins' $B_{A}$ values of each species. We also performed a similar ordinary least squares regression analysis using the PC 1 values derived from the pPCA of our extended 34-taxon dataset to take phylogeny into account. We ran regressions involving pPCA scores twice, once using only our four target species, and once with the 34-taxon dataset.

\section{Results}

\section{Summarized head size measures}

The non-phylogenetically corrected, size-adjusted PCA performed across head measures yielded only a single significant axis (PC 1; Fig. S2). This axis explained $83 \%$ of the total variation in head size data, with all head measures having loaded positively ( $>0.50$; Table S3). The phylogenetically corrected, size-adjusted pPCA yielded similar results. Again, only PC 1 was significant (Fig. S3) and explained $87 \%$ of the total variation in head size data. Each of the head measures loaded positively on PC 1 and had similar magnitudes of covariation between them when correcting for common ancestry $(>0.89$, Table S4).

\section{Relative head size conservatism and ancestral reconstruction}

The Brownian motion model of evolution provided the best fit for our analyses of head size evolution across our phylogeny based on model AICc scores (Table S5). Phylogenetic signal analysis revealed a significant phylogenetic signal was present for head size relative to body size across our tree $(K=0.887$, $P=0.001)$. This result indicates that the sizes of the heads of closely related species resembled each other less than expected under a Brownian motion model of evolution but were more similar to each other than to species drawn from the tree at random. Our reconstruction of relative head sizes (i.e. PC 1) across our phylogeny shows that while several species share similar head morphology to their close relatives, several transitions from small head size to large head size occurred across some lineages (Fig. 1). These transitions were present in all lineages containing bird-egg-eating species in our dataset (i.e. Dispholidus typus, all members of Naja, Pseudaspis cana and Toxicodryas blandingii) but did not occur between Dasypeltis and their ancestors.

\section{Comparisons of head size}

Body size-adjusted head morphology measures were distinctly separated across Dasypeltis and its competitors (Fig. 2). The results of our body size-adjusted MANOVA tests (Homogeneity of slopes test: diet - size: $F_{3,61}=0.122, P=0.947$; sex size: $F_{1}, 63=0.231, P=0.632$ ) confirmed that there were significant differences in average head measures between these species $\left(F_{12,154}=17.352\right.$, Wilk's Lambda $\left.=0.104, P<0.001\right)$ but not between sexes $\left(F_{5,53}=0.463\right.$, Wilk's Lambda $=0.958$, $P=0.802)$ or between the interaction of species and sex $\left(F_{15}\right.$, ${ }_{146}=0.235$, Wilk's Lambda $=0.937, P=0.986$ ). The nonsignificance of sex on the effects of morphological measures suggests that differences in head sizes are not present as a result of sexual size dimorphism.

Overall, Dasypeltis had significantly smaller head dimensions relative to body size than boomslang, cape cobras and mole snakes for all head measures (Fig. 3) as well as for the summarized relative head size derived from PC 1. Dasypeltis had smaller head measures in absolute terms as well (see Table S2). Comparisons between the facultative bird-egg-eating snakes showed that cape cobras differed in head shape to boomslang and mole snakes respectively for several head measures whereas the head measures of boomslang and mole snakes were relatively similar in most cases (Table 1). All four species significantly differed from each other in their dietary niche breadths.

\section{Relationship between dietary niche and head morphology}

Based on our examination of dietary data from the literature, we estimated Levins' measure of niche breadth for the rhombic eggeater as $B=1.00$ (based on 31 feeding records; Bates \& Little, 2013), boomslang as $B=2.91$ (based on 133 feeding records; Smith et al., 2019), cape cobra as $B=4.00$ (based on 101 feeding records; Layloo, Smith \& Maritz, 2017) and mole snake as $B=3.23$ (based on Dyer, 1996; Underhill et al., 2009; and assorted feeding records from various literature and citizen science reports (Maritz \& Maritz, 2020), see Table S6). We standardized these to $B_{A}=0.00$ (rhombic egg-eater), $B_{A}=0.32$ (boomslang), $B_{A}=0.50$ (cape cobra) and $B_{A}=0.47$ (mole snake), respectively (Fig. 4). We also calculated standardized Levin's $B_{A}$ values for our expanded 34-taxon dataset (Table S6).

We found similar results for non-phylogenetically and phylogenetically corrected regression analyses. In both analyses, we 


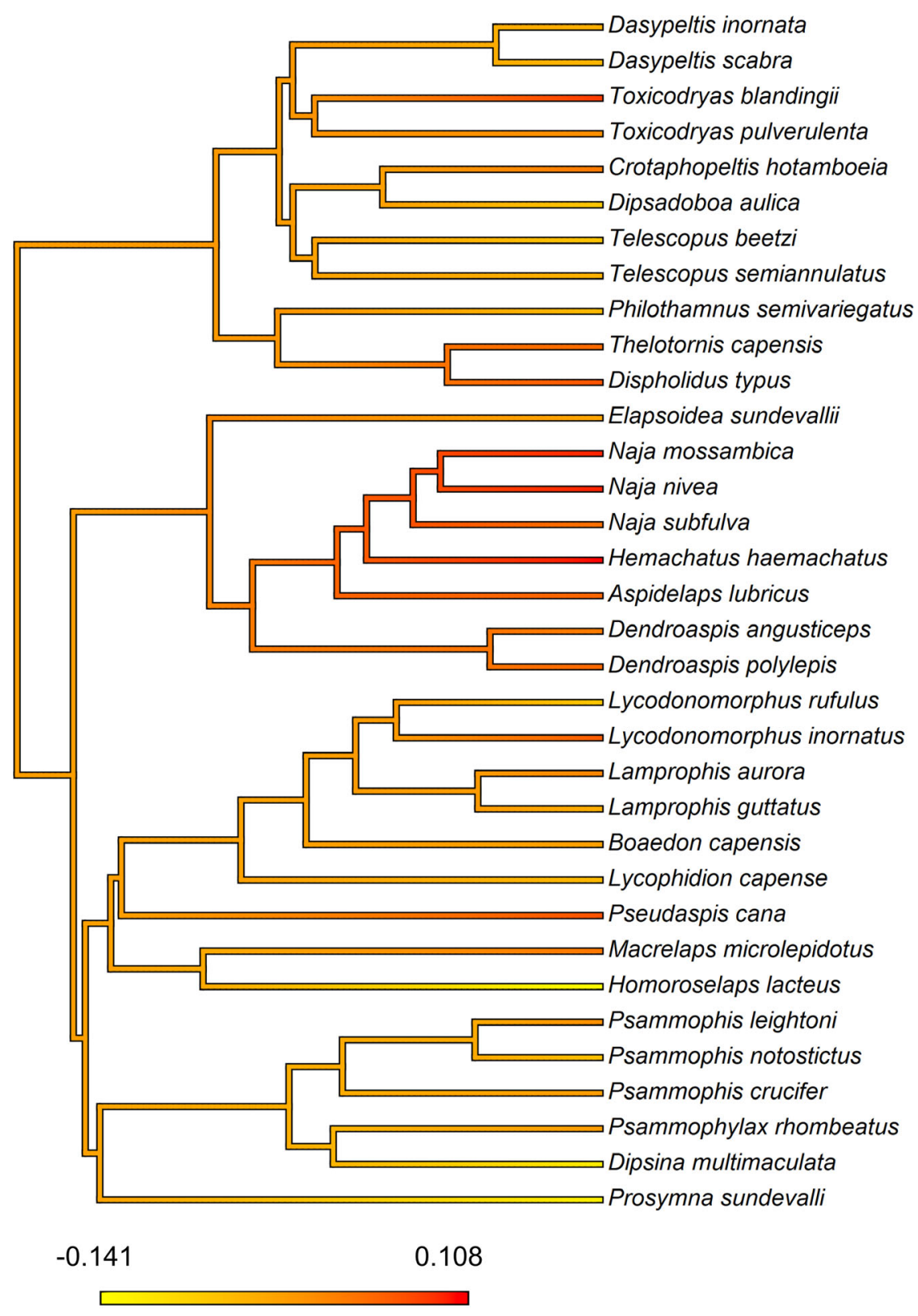

Average scores from PC 1

Figure 1 Ancestral reconstruction of head size covariation relative to body size (PC 1) derived from principal component analyses on sizeadjusted head measures for 34 snake taxa. Yellow = smaller head size, and red =larger head size.

found a significant positive relationship between head sizes and dietary niche breadths across our four study species (nonphylogenetically corrected regression: $F_{1,}{ }_{3}=28.426$, $P=0.003, \beta=0.259, R^{2}=0.934$; phylogenetically corrected regression: $F_{1,3}=76.203, P=0.013, \beta=0.261, R^{2}=0.974$ ) when compared on their own. However, when compared across our expanded 34-taxon dataset, although the relationship remained significant, the correlation between these traits was drastically weaker $\left(F_{1}, \quad 32=4.197, \quad P=0.048, \quad \beta=0.101\right.$, $R^{2}=0.12$; Fig. 5). Overall, dietary specialist species tended to have smaller heads to dietary generalists.

\section{Discussion}

Our results show that contrary to our expectation, rhombic egg-eaters have significantly smaller heads relative to body size 


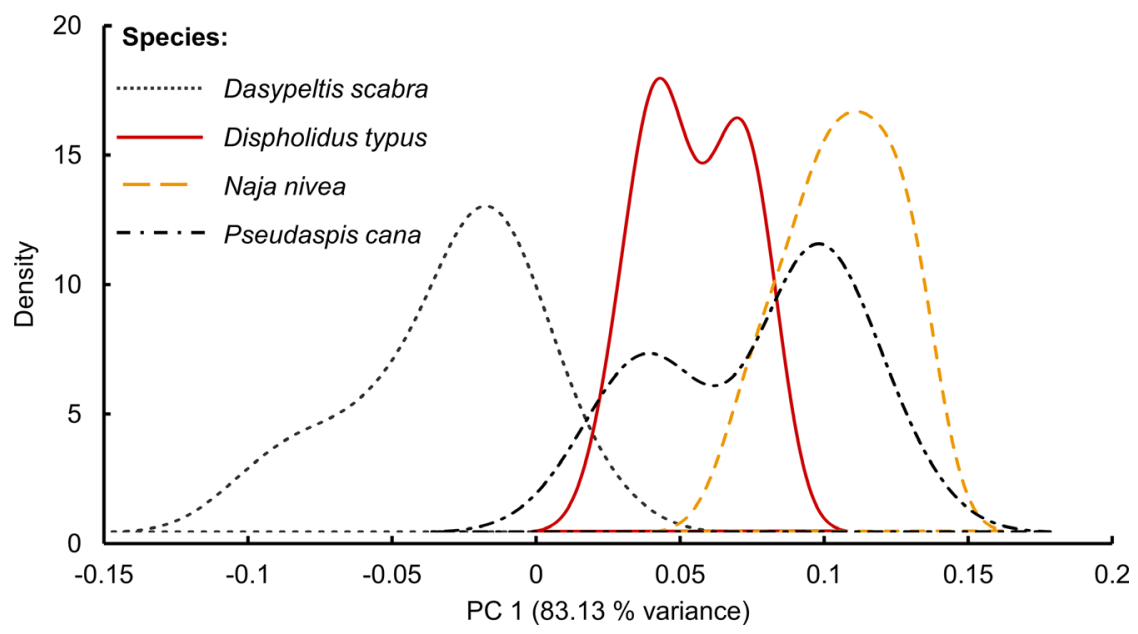

Figure 2 Kernel density estimation of the first PC axis (PC 1) of PCA performed on size-adjusted head measures of our four study species.

than competing boomslang, cape cobras and mole snakes. We found evidence of significant phylogenetic signal present for head size across these taxa and their relatives, suggesting that the disparities in head shapes between them were not present as a result of head size being phylogenetically conserved and were instead derived, possibly as a result of variations in their respective diets. Relative to SVL, an average individual rhombic egg-eater has significantly smaller measures of head height, head length, head width and lower jaw length to equivalently sized competitors in southern Africa. These differences in head size and shape were not affected by variances associated with sexual size dimorphism but were positively associated with dietary niche breadth to some degree, suggesting adaptive responses to feeding were likely selected for as these taxa evolved. Species with the largest heads had the broadest diets, the majority of which included bird eggs.

Dietary specialization in snakes is often associated with adaptive changes in functional morphology, typically in the feeding apparatus, relating to the specific prey type being consumed (Cundall \& Greene, 2000; King, 2002; Hoso, Asami \& Michio, 2007). Typically, snakes that have adapted to consume bulky, robust prey types possess long head and jaw elements and have relatively large gapes that allow them to efficiently exploit large prey (Arnold, 1993; Jayne, Voris \& Ng, 2018; Moon et al., 2019). Given the findings of de Queiroz \& Rodríguez-Robles, (2006) regarding the challenges of consuming bird eggs, we expected to find that specialist egg-eaters would have relatively large heads that allow them to compete with the absolutely larger heads of their large-bodied competitors, but this was not the case. Instead, we found that eggeaters have smaller heads than other southern African snakes that facultatively bird eggs. Moreover, our ancestral reconstruction of head size across this phylogeny showed that the large head sizes of facultative bird-egg-eating species evolved from ancestors with smaller sized heads, but that this transition did not take place within the Dasypeltis lineage. The evolution of large head size in those species may therefore have resulted from a variety of environmental factors unrelated to the consumption of bird eggs.

Interspecific competition for food by snakes typically occurs between species that share similarities in their ecologies, morphologies and ancestry (for examples see Luiselli, 2006; Luiselli, 2008; Maritz, Alexander \& Maritz, 2019). In Dasypeltis, the development of key phenotypical innovations and functional changes within their feeding apparatus allow these snakes to easily ingest bird eggs, a source of prey that most of their relatives are unable to consume. At present, egg-eaters do not compete with similarly sized closely related genera, like Crotaphopeltis or Dipsadoboa, and instead share and compete over food resources with phylogenetically distantly related species that morphologically differ from themselves in both absolute and relative morphology. Consequently, the specialized adaptations of egg-eaters, including their highly flexible jaw and neck tissue and vertebral hypapophyses (Gans, 1952), allow for the decoupling of typical body-size-constrained competition dynamics within at least some southern African snake communities. This represents a clear example of how the evolution of unique phenotypic traits can alter species interactions and community dynamics, the results of which can modify the relationship between phylogenetic distance and species interaction strength among dissimilar species within evolving metacommunities (Hunter, 1998; Nascimento et al., 2011; Weber et al., 2017).

Competition between Dasypeltis and facultative bird-eggeating snakes in contemporary systems is potentially present as a consequence of historical competition among ancestral lineages, in line with the theory of the ghost of competition past (Weber et al., 2017; Ye, Yang \& Liang, 2019). Before adapting to exclusively feeding on bird eggs, ancestral Dasypeltis likely had a broader dietary niche (de Queiroz \& Rodríguez-Robles, 2006) and probably competed with several snake species of varying sizes. The transition towards bird egg specialization by Dasypeltis may have been selected in response to competition for non-avian prey by ancestral colubrids and other 

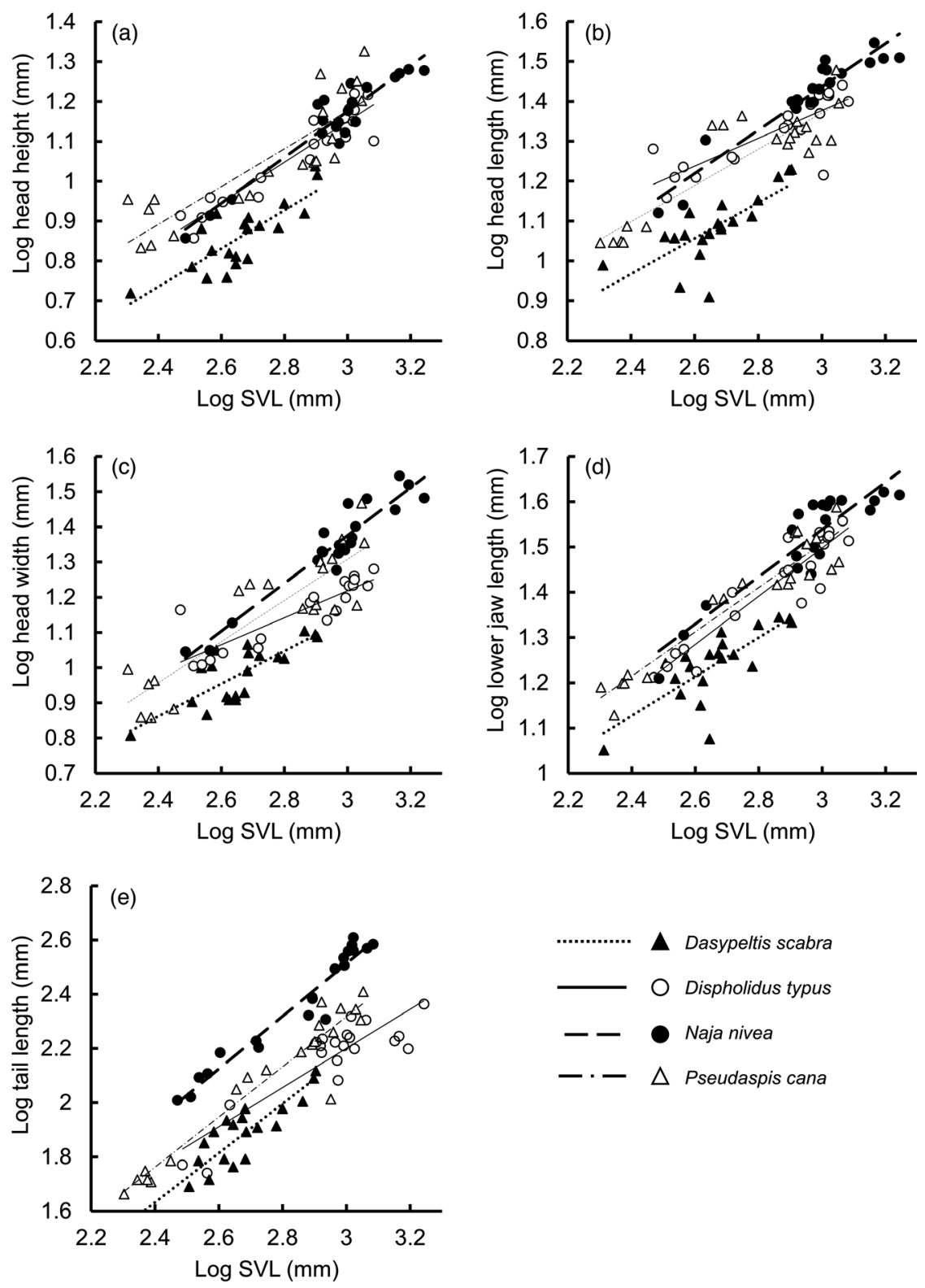

Figure 3 Relationships between (a) log head height, (b) log head length, (c) log head width, (d) log lower jaw length and (e) log tail length relative to log SVL for Dasypeltis scabra, Dispholidus typus, Naja nivea and Pseudaspis cana specimens measured in this study.

ecologically similar African snakes as a result of resource partitioning. If so, this divergence may have led to a reduction of competition for Dasypeltis by transitioning from a competitive network involving several small species of snakes to one comprised of fewer but larger snake species that greatly differ from Dasypeltis in their morphology and ecology.

Variation in the dietary compositions and the degree of dietary specialization versus generalization in snakes could explain the similarities and differences in head morphology between the facultative egg-eating snakes in our comparison (Arnold, 1993; Greene, 1997; Cundall \& Greene, 2000; Moon et al., 2019). Boomslang, cape cobras and mole snakes each vary in their utilization of different prey resources, and these differences appear to conform with the disparities between their assorted head morphologies. Cape cobras occupied the most extreme position of morphological space in our analyses of head size covariation and had significantly larger head measures to boomslang and mole snakes, respectively. Cape cobras have the broadest dietary niche of the four species and are largely ophiophagous (Maritz, Alexander \& Maritz, 2019). The consumption of robust, elongate prey types presents a unique set of mechanical challenges of ingestion not present in boomslang and mole snakes. Differences in head size between boomslang and mole snakes were less prominent, although 
Table 1 Pairwise mean differences ( $P$-values in parenthesis) for species-level comparisons of size-adjusted head measures, PC axes and dietary niche breadths for four competing bird-egg-eating snake species

\begin{tabular}{|c|c|c|c|c|}
\hline & & Dasypeltis scabra & Dispholidus typus & Naja nivea \\
\hline \multirow[t]{4}{*}{ Head height } & D. scabra & - & & \\
\hline & D. typus & $0.056(<0.001)$ & - & \\
\hline & N. nivea & $0.070(<0.001)$ & $0.014(0.368)$ & - \\
\hline & Pseudaspis cana & $0.061(<0.001)$ & $0.005(0.999)$ & 0.009 (0.999) \\
\hline \multirow[t]{4}{*}{ Head length } & D. scabra & - & & \\
\hline & D. typus & $0.055(<0.001)$ & - & \\
\hline & N. nivea & $0.074(<0.001)$ & $0.019(0.048)$ & - \\
\hline & P. cana & $0.055(<0.001)$ & $0.001(0.999)$ & $0.018(0.066)$ \\
\hline \multirow[t]{4}{*}{ Head width } & D. scabra & - & & \\
\hline & D. typus & $0.035(<0.001)$ & - & \\
\hline & N. nivea & $0.090(<0.001)$ & $0.055(<0.001)$ & - \\
\hline & P. cana & $0.063(<0.001)$ & $0.028(0.003)$ & $0.027(0.004)$ \\
\hline \multirow[t]{4}{*}{ Lower jaw length } & D. scabra & - & & \\
\hline & D. typus & $0.034(<0.001)$ & - & \\
\hline & N. nivea & $0.048(<0.001)$ & $0.014(0.196)$ & - \\
\hline & P. cana & $0.038(<0.001)$ & $0.004(0.999)$ & $0.009(0.905)$ \\
\hline \multirow[t]{4}{*}{ PCA PC1 } & D. scabra & - & & \\
\hline & D. typus & $0.089(<0.001)$ & - & \\
\hline & N. nivea & $0.142(<0.001)$ & $0.053(0.001)$ & - \\
\hline & P. cana & $0.110(<0.001)$ & $0.020(0.296)$ & $0.032(0.017)$ \\
\hline \multirow[t]{4}{*}{ Levin's BA } & D. scabra & - & & \\
\hline & D. typus & $0.317(<0.001)$ & - & \\
\hline & N. nivea & $0.499(<0.001)$ & $0.182(<0.001)$ & - \\
\hline & P. cana & $0.469(<0.001)$ & $0.153(<0.001)$ & $0.030(<0.001)$ \\
\hline
\end{tabular}

Significant values in boldface.

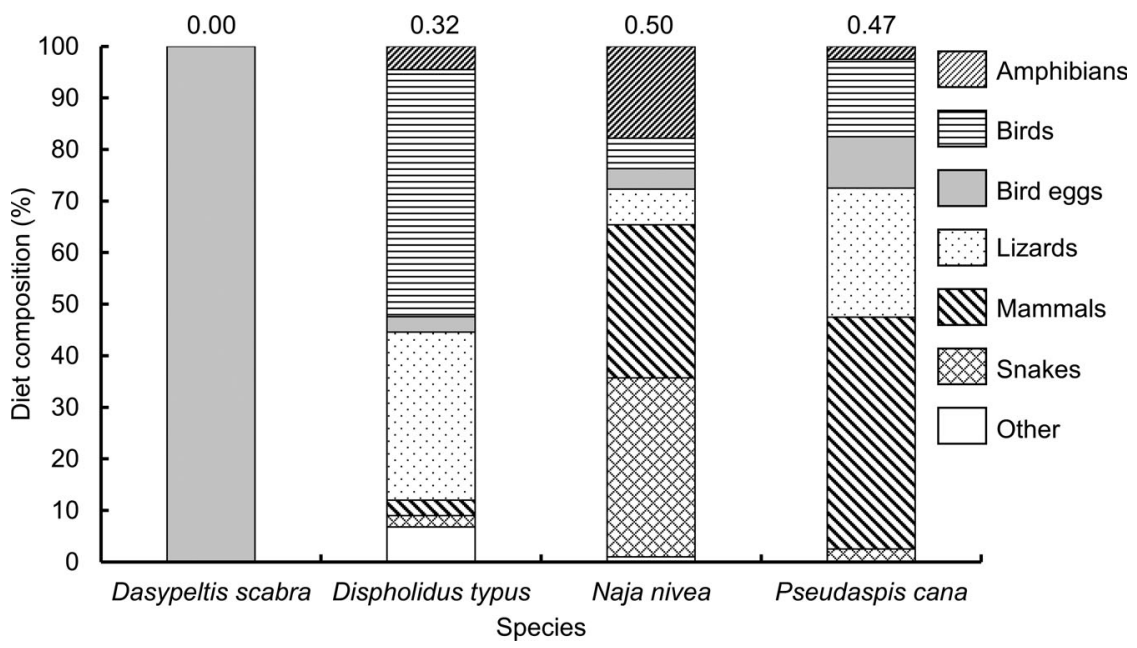

Figure 4 Diet compositions as estimated from literature sources for Dasypeltis scabra, Dispholidus typus, Naja nivea and Pseudaspis cana. Data labels above bars represent standardized measures of Levins' Niche Breadth $\left(B_{A}\right)$.

mole snakes have wider heads, possibly reflecting their propensity for preying on mammals and adult birds (Maritz \& Maritz, 2020) whereas boomslang primarily consumes chicks, nestlings and chameleons (Smith et al., 2019).

Field studies investigating competitive interactions between egg-eaters and their rivals are scarce, but laboratory-based experiments have shown that egg-eaters are extremely proficient at exploiting bird eggs despite their small body size. Gartner \& Greene, (2008) compared the feeding performance of Dasypeltis to a facultative bird-egg-eating species from North America, the common king snake, Lampropeltis getula and found that only large king snakes could consume small eggs while equivalently sized Dasypeltis showed much greater ingestion ability. Moreover, Gartner \& Greene, (2008) suggest 


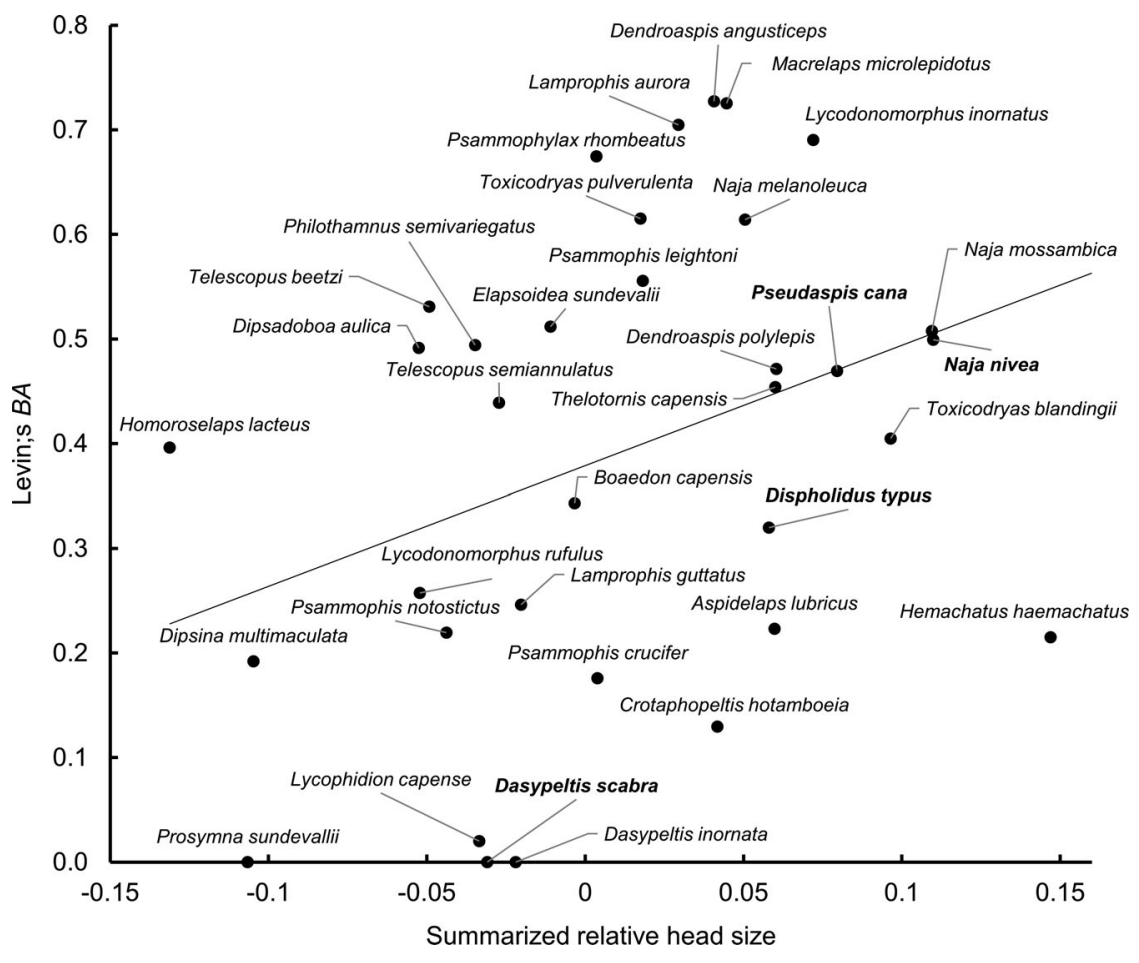

Figure 5 Relationship between summarized head sizes derived from phylogenetic PCA, and Levins' standardized measures of niche breadth between snake species in our expanded 34-taxon dataset. Our four target species are highlighted in boldface.

that adult egg-eaters are likely able to consume up to $98 \%$ of bird eggs that they encounter across their range within southern Africa. Egg-eaters may have therefore adapted towards a maximum head size optimum concomitant with the maximum sizes of available bird eggs which could potentially explain their small size. The apparent mismatch in egg-eater head size and bird egg-ingestion ability is largely achieved through the high degree of stretchability in the necks and jaws of these snakes, the limits of which require further investigation. A detailed examination of bird-egg-eating performance and flexibility in gape size in egg-eater's direct competitors could provide further insight into competition for bird eggs between snakes in Africa.

For most snakes, head size is a major contributing factor towards determining the suite of prey that individuals can consume. However, several studies have shown that in some species, head morphology exhibits phenotypic plasticity in response to prey size and feeding opportunities. For example, Aubret, Shine \& Bonnet (2004) found that tiger snakes (Notechis scutatus) on mainland Australia have smaller heads than individuals from populations on nearby islands and attributed these differences to adaptive plasticity in response to differences in diet between those populations. Queral-Regil \& King (1998) found that different feeding regimes affected head size development in North American water snakes (Nerodia sipodon). Similarly, Bonnet et al., (2001) saw similar trends in head size development in captive gaboon adders (Bitis gabonica) from Africa. Plasticity in head size in response to prey size does not appear to be present in all species of snakes (see Forsman, 1996; Schuett et al., 2005) and whether this is present in our study species requires clarification. Adult mole snakes vary in size from 1.4 to $2 \mathrm{~m}$, with individuals from the Western Cape being particularly large. Similarly, adult boomslang varies between 1.2 and $2 \mathrm{~m}$, and cape cobras vary between 1.4 and $2.3 \mathrm{~m}$ (Alexander \& Marias 2007). Whether these intraspecific difference in body size, and therefore also head size, manifest as a result of plastic responses to variable prey intake, other environmental factors, or are entirely genetic is unclear.

Although prey size is a major factor in influencing head shape within most snakes (Arnold, 1993; Cundall \& Greene, 2000; Jayne, Voris \& Ng, 2018; Segall et al., 2020), the link between prey size and head size possibly only accounts for challenges relating to ingestion (Vincent et al., 2006). It is important to also consider other selective pressures that may have been involved in the evolution of head shape for different snake species. Factors such as habitat use, prey capture and anti-predator defensive behaviour can play prominent roles in the evolution of the feeding apparatus and head sizes of many snake species (Hibbits \& Fitzgerald, 2005; Fabre et al., 2016; Segall et al., 2020). For example, the defensive display of Dasypeltis involves flattening and triangulation of their heads which they achieve by manipulating their quadrates (Young, Lalor \& Solomon, 1999), a feature strongly associated with gape size (King, 2002; Moon et al., 2019). However, while the evolution of head triangulation may have affected jaw 
structure and head size within Dasypeltis, it is thought to have evolved after their lineage adopted their dietary specialist lifestyles (Gans \& Richmond, 1957).

\section{Conclusion}

Our study has important implications for understanding ecological interactions between competing snake species within and beyond the southern African region. Our findings suggest that specialized adaptations can decouple typical body-sizeconstrained competition dynamics between sympatric snake species derived from ancient selective pressures. Competition for specific food resources between snakes may be present within distantly related, phenotypically dissimilar lineages today as a consequence of resource partitioning among ancestor species. We suggest that to understand competition within contemporary snake communities, researchers should seek to also explore shifts in dietary niche utilization within extant snakes and their ancestors.

\section{Acknowledgements}

We thank W. Conradie and the Bayworld Museum as well as J. Opperman and the Iziko South African Museum for allowing the use of specimens for this study. We thank the reviewers for their valuable insights and comments on an earlier version of this work. We also thank the University of the Western Cape and the National Research Foundation for providing funding that facilitated this study (NRF UIDs:118090 \& 123281).

\section{References}

Akaike, H. (1973). Information theory and an extension of the maximum likelihood principle. In Second International Symposium on Information Theory: 267-281. Csaki, F. (Ed.). Budapest: Akademiai Kiado.

Alatalo, R.V. \& Moreno, J. (1987). Body size, interspecific interactions, and use of foraging sites in tits (Paridae). Ecology 68, 1773-1777.

Alexander, G.J. \& Marias, J. (2007). A guide to the reptiles of southern Africa. Cape Town: Penguin Random House South Africa.

Arnold, S.J. (1993). Foraging theory and prey-size-predator-size relations in snakes. In Snakes: ecology and behaviour: 87115. Seigel, R.A. \& Collins, J.T. (Eds.). New York: McGrawHill.

Arthur, W. (1987). The niche in competition and evolution. New York: John Wiley \& Sons.

Aubret, F., Shine, R. \& Bonnet, X. (2004). Adaptive developmental plasticity in snakes. Nature 431, 261-262.

Bates, M.F. \& Broadley, D.G. (2018). A revision of the eggeating snakes of the genus Dasypeltis Wagler (Squamata: Colubridae: Colubrinae) in north-eastern Africa and southWestern Arabia, with descriptions of three new species. Indago 34, 1-95.
Bates, M.F. \& Little, I.T. (2013). Predation on the eggs of ground-nesting birds by Dasypeltis scabra (Linnaeus, 1758) in the moist highland grasslands of South Africa. Afr. J.

Herpetol. 62, 125-134.

Bloch, C.P., Stevens, R.D. \& Willig, M.R. (2011). Body size and resource competition in New World bats: a test of spatial scaling laws. Ecography 34, 460-468.

Blomberg, S.P., Garland Jr, T. \& Ives, A.R. (2003). Testing for phylogenetic signal in comparative data: behavioral traits are more labile. Evolution 57, 717-745.

Bonnet, X., Shine, R., Naulleau, G. \& Thiburce, C. (2001). Plastic vipers: influence of food intake on the size and shape of Gaboon vipers (Bitis gabonica). J. Zool. (Lond.) 255, 341351.

Branch, B. (1998). Field guide to snakes and other reptiles of southern Africa. Cape Town: Struik Publishers.

Broadley, D.G. (1990). Fitzsimmons'snakes of southern Africa. Johannesburg: Delta Books.

Case, T.J., Faaborg, J. \& Sidell, R. (1983). The role of body size in the assembly of West Indian bird communities. Evolution 37, 1062-1074.

Cundall, D. (2019). A few puzzles in the evolution of feeding mechanisms in snakes. Herpetologica 75, 99-107.

Cundall, D. \& Greene, H.W. (2000). Feeding in snakes. In Feeding: form, function, and evolution in tetrapod vertebrates: 293-333. Schwenk, K. (Ed.). London: Academic Press.

de Queiroz, A. \& Rodríguez-Robles, J.A. (2006). Historical contingency and animal diets: the origins of egg eating in snakes. Am. Nat. 167, 684-694.

Dyer, B.M. (1996). Predation by snakes on seabirds at three South African islands. Afr. J. Mar. Sci. 17, 309-313.

Fabre, A.C., Bickford, D., Segall, M. \& Herrel, A. (2016). The impact of diet, habitat use, and behaviour on head shape evolution in homalopsid snakes. Biol. J. Linn. Soc. 118, 634-647.

Forsman, A. (1996). An experimental test for food effects on head size allometry in juvenile snakes. Evolution 50, 2536-2542.

Gans, C. (1952). The functional morphology of the egg-eating adaptations in the snake genus Dasypeltis. Zoologica 37, 209244.

Gans, C. (1974). Biomechanics: an approach to vertebrate biology. Michigan: University of Michigan Press.

Gans, C. \& Richmond, N.D. (1957). Warning behaviour in snakes of the genus Dasypeltis. Copeia 4, 269-274.

Gartner, G.E.A. \& Greene, H.W. (2008). Adaptation in the African egg-eating snake: a comparative approach to a classic study in evolutionary functional morphology. J. Zool. (Lond.) 275, 368-374.

Greene, H.W. (1997). Snakes: The evolution of mystery in nature. Berkeley: University of California Press.

Hibbits, T.J. \& Fitzgerald, L.A. (2005). Morphological and ecological convergence in two natricine snakes. Biol. J. Linn. Soc. 85, 363-371.

Hoso, M., Asami, T. \& Michio, H. (2007). Right-handed snakes: convergent evolution of asymmetry for functional specialization. Biol. Lett. 3, 169-172. 
Hunter, J.P. (1998). Key innovations and the ecology of macroevolution. Trends. Ecol. 13, 31-36.

IBM Corp (2017). IBM SPSS Statistics for Windows. Version 23. New York: IBM Corp.

Jackson, D.A. (1993). Stopping rules in principal components analysis: a comparison of heuristical and statistical approaches. Ecology 74, 2204-2214.

Jayne, B.C., Voris, H.K. \& Ng, P.K. (2018). How big is too big? Using crustacean-eating snakes (Homalopsidae) to test how anatomy and behaviour affect prey size and feeding performance. Biol. J. Linn. Soc. 123, 636-650.

King, R.B. (2002). Predicted and observed maximum prey sizesnake size allometry. Funct. Ecol. 16, 766-772.

LaBarbera, M. (1989). Analyzing body size as a factor in ecology and evolution. Annu. Rev. Ecol. Systemat. 20, 97-117.

Layloo, I., Smith, C.C.D. \& Maritz, B. (2017). Diet and feeding in the Cape Cobra, Naja nivea. Afr. J. Herpetol. 66, 147-153.

Levins, R. (1968). Evolution in changing environments: some theoretical explorations. Princeton: Princeton University Press.

Luiselli, L. (2006). Resource partitioning and interspecific competition in snakes: the search for general geographical and guild patterns. Oikos 114, 193-211.

Luiselli, L. (2008). Community ecology of African reptiles: historical perspective and a meta-analysis using null models. Afr. J. Ecol. 46, 384-394.

Maritz, B., Alexander, G.J. \& Maritz, R.A. (2019). The underappreciated extent of cannibalism and ophiophagy in African cobras. Ecology 100, e02522.

Maritz, R.A. \& Maritz, B. (2020). Sharing for Science: highresolution trophic interactions revealed rapidly by social media. PeerJ 8, e9485.

Mcgill, B., Enquist, B., Weiher, E. \& Westoby, M. (2006). Rebuilding community ecology from functional traits. Trends. Ecol. 21, 178-185.

Moon, B.R., Penning, D.A., Segall, M. \& Herrel, A. (2019). Feeding in snakes: form, function, and evolution of the feeding system. In Feeding in vertebrates: evolution, morphology, behaviour, biomechanics: 527-574. Bels, V. \& Whishaw, I.Q. (Eds.). Cham: Springer Nature.

Mori, A. \& Vincent, S.E. (2008). An integrative approach to specialization: relationships among feeding morphology, mechanics, behaviour, performance and diet in two syntopic snakes. J. Zool. (Lond.) 275, 47-56.

Morin, P.J. \& Johnson, E.A. (1988). Experimental studies of asymmetric competition among anurans. Oikos 53, 398-407.

Nakayama, S. \& Fuiman, L.A. (2010). Body size and vigilance mediate asymmetric interference competition for food in fish larvae. Behav. Ecol. 21, 708-713.

Nascimento, F.J.A., Karlson, A.M.L., Näslund, J. \& Elmgren, R. (2011). Diversity of larger consumers enhances interference competition effects on smaller competitors. Oecologia 166, 337-434.

Pennell, M.W., Eastman, J.M., Slater, G.J., Brown, J.W., Uyeda, J.C., FitzJohn, R.G., Alfaro, M.E. \& Harmon, L.J. (2014).
Geiger v2. 0: an expanded suite of methods for fitting macroevolutionary models to phylogenetic trees.

Bioinformatics 30, 2216-2218.

Persson, L. (1985). Asymmetrical competition: are larger animals competitively superior? Am. Nat. 126, 261-266.

Pianka, E.R. (1978). Evolutionary ecology (2nd edn). New York: Harper \& Row.

Pyron, R.A., Burbrink, F.T. \& Wiens, J.J. (2013). A phylogeny and revised classification of Squamata, including 4161 species of lizards and snakes. BMC Evol. Biol. 13, 1-54.

Queral-Regil, A. \& King, R.B. (1998). Evidence for phenotypic plasticity in snake body size and relative head dimensions in response to amount and size of prey. Copeia 1998, 423-429.

$\mathrm{R}$ Core Team (2020). $R$ : a language and environment for statistical computing. Vienna: R Foundation 403 for Statistical Computing.

Ramsay, K., Kaiser, M.J. \& Hughes, R.N. (1997). A field study of intraspecific competition for food in hermit crabs (Pagurus bernhardus). Estuar. Coast. Shelf Sci. 44, 213-220.

Revell, L.J. (2012). phytools: an R package for phylogenetic comparative biology (and other things). Methods Ecol. Evol. 3, 217-223.

Robertson, D.R. (1998). Implications of body size for interspecific interactions and assemblage organization among coral-reef fishes. Austral Ecol. 23, 252-257.

Rodríguez-Robles, J.A., Bell, C.J. \& Greene, H.W. (1999). Gape size and evolution of diet in snakes: feeding ecology of erycine boas. J. Zool. (Lond.) 248, 49-58.

Roughgarden, J. (1983). Competition and theory in community ecology. Am. Nat. 122, 583-601.

Schoener, T.W. (1974). Resource partitioning in ecological communities. Science 185, 27-39.

Schoener, T.W. (1983). Field experiments on interspecific competition. Am. Nat. 122, 240-285.

Schuett, G.W., Hardy, D.L., Earley, R.L. \& Greene, H.W. (2005). Does prey size induce head skeleton phenotypic plasticity during early ontogeny in the snake Boa constrictor? J. Zool. (Lond.) 267, 363-369.

Segall, M., Cornette, R., Godoy-Diana, R. \& Herrel, A. (2020). Exploring the functional meaning of head shape disparity in aquatic snakes. Ecol. Evol. 10, 6993-7005.

Shine, R. (1991). Why do larger snakes eat larger prey items? Funct. Ecol. 5, 493-502.

Shine, R., Branch, W.R., Webb, K.K., Harlow, P.S. \& Shine, T. (2006). Sexual dimorphism, reproductive biology, and dietary habits of Psammophiine snakes (Colubridae) from southern Africa. Copeia 2006, 650-664.

Smith, C.C.D., Layloo, I., Maritz, R.A. \& Maritz, B. (2019). Sexual dichromatism does not translate into sex-based difference in morphology or diet for the African boomslang. J. Zool. (Lond.) 308, 253-258.

Tarboton, W.R. (2011). Guide to the nests and eggs of southern African birds. Cape Town: Struik Publishers.

Toft, C.A. (1985). Resource partitioning in amphibians and reptiles. Copeia 1985, 1-21. 
Travis, J. (1980). Phenotypic variation and the outcome of interspecific competition in hylid tadpoles. Evolution 34, 4050.

Underhill, L.G., Sherley, R.B., Dyer, B.M. \& Crawford, J.M. (2009). Interactions between snakes and seabirds on Robben, Schaapen and Meeuw Islands, Western Cape province, South Africa. Ostrich 80, 115-118.

Vincent, S.E., Brandley, M.C., Herrel, A. \& Alfaro, M.E. (2009). Convergence in trophic morphology and feeding performance among piscivorous natricine snakes. J. Evol. Biol. 22, 1203-1211.

Vincent, S.E., Dang, P.D., Herrel, A. \& Kley, N.J. (2006). Morphological integration and adaptation in the snake feeding system: a comparative phylogenetic study. J. Evol. Biol. 19, 1545-1554.

Weber, M.G., Wagner, C.E., Best, R.J., Harmon, L.J. \& Matthews, B. (2017). Evolution in a community context: on integrating ecological interactions and macroevolution. Trends. Ecol. 32, 291-304.

Ye, P., Yang, C. \& Liang, W. (2019). Nest site availability and niche differentiation between two cavity-nesting birds in time and space. Ecol. Evol. 2019, 11904-11910.

Young, B.A., Lalor, J. \& Solomon, J. (1999). The comparative biomechanics of an ophidian defensive behaviour: head triangulation in hognose snake (Heterodon) and an egg-eating snake (Dasypeltis). J. Zool. (Lond.) 248, 169-177.

Zeng, X.H. \& Lu, X. (2009). Interspecific dominance and asymmetric competition with respect to nesting habitats between two snowfinch species in a high-altitude extreme environment. Ecol. Res. 24, 607-616.

Zheng, Y. \& Wiens, J.J. (2016). Combining phylogenomic and supermatrix approaches, and a time-calibrated phylogeny for squamate reptiles (lizards and snakes) based on 52 genes and 4162 species. Mol. Phylogenetics. Evol. 94, 537-547.

\section{Supporting Information}

Additional Supporting Information may be found in the online version of this article:

Appendix S1. Figure S1. Phylogeny of our four study species and selected closely related snake taxa from the Colubridae, Elapidae, and Lamprohiidae sensu lato families respectively derived from the phylogeny of squamate reptiles produced in Zheng \& Wiens, (2016).

Figure S2. Scree plot of non-phylogenetic PCA of body size-adjusted head measures.

Figure S3. Scree plot of phylogenetic PCA of body size-adjusted head measures.

Table S1. List of voucher numbers for measured specimens. PEM-R denotes Bayworld Museum, SAM ZR denotes Iziko South African Museum, and UWC denotes the University of the Western Cape.

Table S2. Mean \pm SD morphological measurements of study specimens ( $\min$ and max in parenthesis). All measurements in $\mathrm{mm}$.

Table S3. Non-phylogenetic variance-covariance matrix of size-adjusted head size measures used in this study.

Table S4. Phylogenetic variance-covariance matrix of sizeadjusted head size measures used in this study.

Table S5. Summary of evolutionary model tests for relative head size across our 34-taxon phylogeny.

Table S6. Proportions of dietary resource use and dietary niche breadths for our expanded 34-taxon dataset. 